\title{
NOTE ON REGURRENT DISLOCATION OF THE SHOULDER JOINT
}

\section{Superior Approach Causing the Only Failure in Fifty-two Operations for Repair of the Labrum and Capsule}

\author{
Reginald Watson-Jones, London, England
}

The clinical records of seventy-one patients on whom I have operated for recurrent dislocation of the shoulder joint have been re-examined. They may be analysed as follows:

$\begin{array}{lccc}\text { Fascial sling operation (Henderson) . . . } & \text { Cases } & \text { Recurrences } \\ \text { Transplantation of biceps tendon (Heymanowitsch, Nicola) } & 1 & 1 \\ \text { Labrum repair and capsular reefing (Bankart, Putti, Platt) } & \mathbf{5 2} & 5\end{array}$

Fascial sling operation-The single case of fascial slinging, performed many years ago by the Henderson technique, may be dismissed; the operation is known to be unreliable and it has long since been abandoned.

Transplantation of biceps tendon-The Nicola operation with its various modifications has also been abandoned for more than five years. In this particular series the failed cases were shown at secondary operation to have sustained rupture of the transplanted tendon. This is by no means true of all failures of the Nicola transplant. The operation may fail for a number of reasons, and notably because the biceps tendon is often so placed that the humeral head can rotate around it and dislocate without so much as a strain being thrown upon the tendon. The explanation of the constancy of tendon rupture in this series possibly lies in the fact that the humerus was always drilled while it was held in the position of full internal rotation, and the direction of the drill hole was so planned that the tendon served as a check to external rotation movement which has long been recognised as the exciting cause of recurrent dislocation. Such check may explain the success of the operation in a proportion of patients, particularly those who are prepared to lead a sedentary life: But in this series of young athletic men it failed in one-third of the cases. Whatever the cause of failure, whether through misplacement of the tendon, or through rupture of the tendon because it is unequal to the task, the operation fails far too often.

Repair of glenoid labrum with reefing of capsule--The particular purpose of this note is to record fifty-two operations in which the glenoid labrum was exposed. In fifiy-one operations an anterior exposure was used: if the labrum was detached it was resutured; in any event the anterior capsule was plicated and the subscapularis shortened; and all fifty-one operations succeeded in preventing recurrence. In one operation a superior exposure was used: an extensively detached labrum was securely stitched to bone; but the anterior capsule and subscapularis were never divided; indeed they were never exposed; they were not reefed or shortened; and the operation failed, dislocation recurring within six months.

In each of the fifty-one cases Bankart's exposure was followed in every detail--the deltoid and pectoralis major were separated, the coracoid process osteotomised, the coracobrachialis and biceps retracted downwards, the subscapularis divided and retracted inwards, and the capsule divided to expose the glenoid margin. In thirty-six of the fifty-one cases (70 per cent.) there was a definite lesion of the labrum, ranging from separation in its lower part to bucket-handle displacement across the joint. In seven cases it was recorded at the time of operation that "there was a doubtful lesion," and in eight cases there was no lesion at all. Damage to the head of the humerus varied from simple bruising of articular cartilage in the postero-lateral sector, through every stage of "osteochondritis dissecans" to excavation of as much as one-third of the bone.

When the labrum was detached it was repaired by the Bankart technique. When the capsule was detached from the labrum, the labrum was usually resected and the capsule 


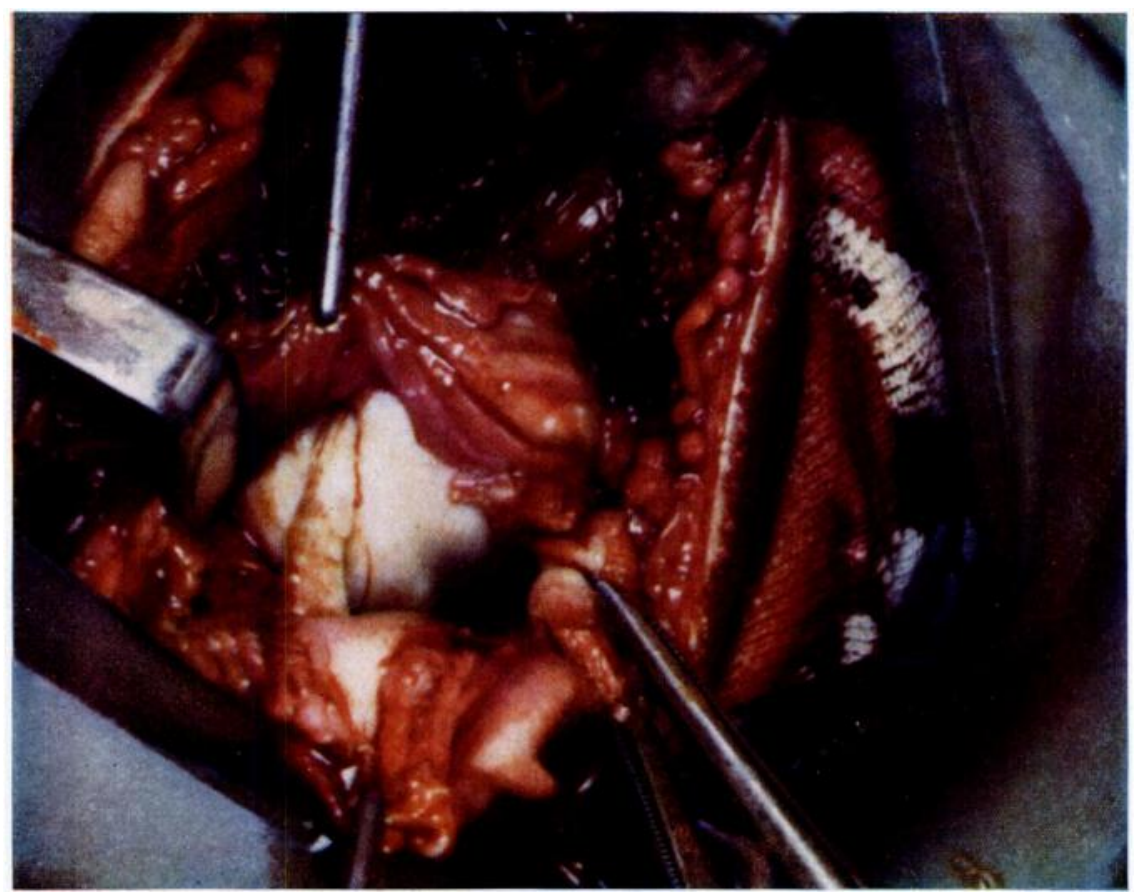

Fig. 1

Recurrent dislocation of right shoulder joint exposed by superior approach showing : 1) transplanted biceps tendon which is intact, but is not controlling external rotation movement or preventing redislocation; 2) large defect in the postero-lateral sector of the head of the humerus.

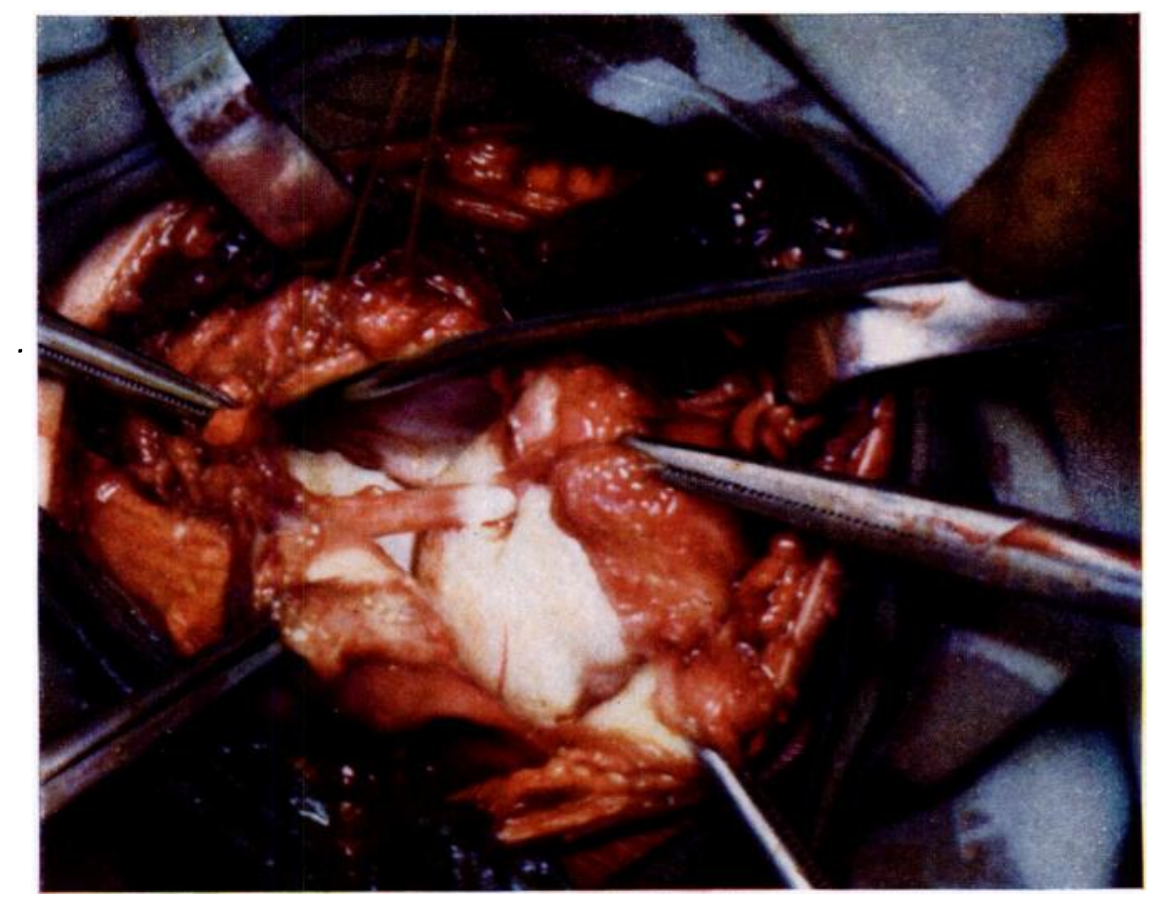

Fig. 2

When the head of the humerus was pulled outwards and backwards an unusually clear view of the anterior glenoid margin was gained. The widely detached labrum and capsule are being lifted by an elevator to show the large pocket into which the defective head of the humerus could subluxate. 
sutured to bone. But in every case, whether or not there was a labrial defect capable of repair, the wound was closed with overlapping and double-breasting of capsule and slight shortening of the subscapularis by stitching it to a more distal insertion in the humerus-the procedure formerly known as capsulorrhaphy, and now described in a slightly modified form as the Putti-Platt operation. All these cases were successful, and so far as is known there has been no recurrence.

The one case which is particularly instructive is shown in Figs. 1-3. For a number of reasons it was decided to expose the joint by means of a superior approach. The operation note, dictated by $\mathrm{W}$. McKecknie who at that time was my first assistant, reads as follows:

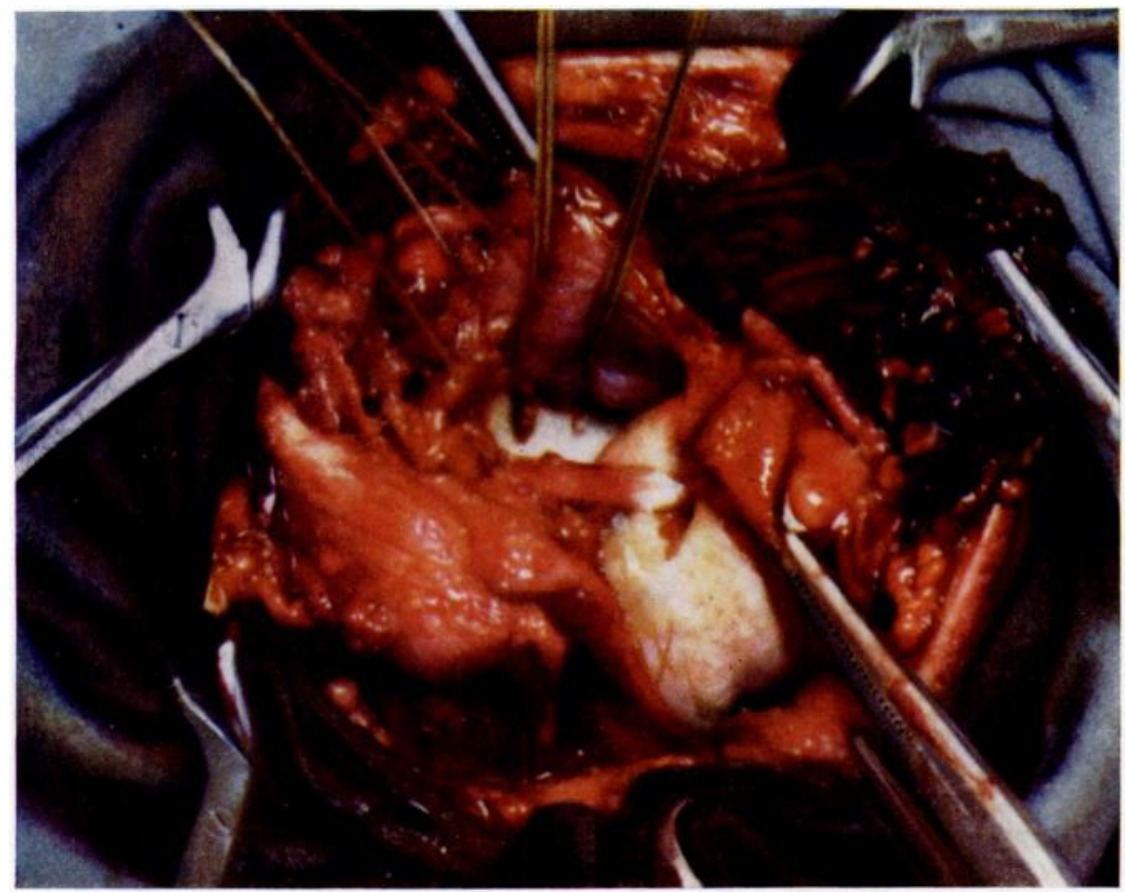

IIIG. 3

With the head of the humerus still retracted outwards and backwards two strong sutures have been inserted through the anterior glenoid margin and the detached labrum and capsule. The sutures are ready for tying.

Operation, November 15, 1945--Sabre-blade approach to right shoulder by four-inch incision centred on the acromio-clavicular joint. Ostentomy of base of the acromion which was reflected outwards together with the clavicular origin of the deltoid. Longitudinal incision in the line of fibres of the musculotendinous cuff, with dissection of the anterior flap from the front of the tuberosity to expose the humeral head and glenoid from above. This revealed: $(a)$ a large deficiency in the posterior aspect of the humeral head, well rounded, and covered with fibro-cartilage; $(b)$ tendon of the biceps, previously transplanted by Nicola operation performed elsewhere, passing to the head of the humerus with no solution of continuity: it was quite loose and in no way held the head of the humerus or prevented it dislocating; in fact the proximal glenoid attachment was increasing displacement of the labrum by pulling it across the joint like a bucket-handle tear of a knee joint cartilage; (c) complete detachment of the glenoid labrum from bone; (d) a large pocket in front of the glenoid measuring two inches in diameter. The front of the neck of the scapula and the glenoid margin were freshened with an osteotome. Double holes were drilled, and the labrum and capsule were securely stitched to bone by strong nylon and silkworm sutures.

Observations at the time of operation--(1) As an operative exposure this would seem to have many advantages over the anterior approach: (a) there is less muscle splitting and division; $(b)$ there is no disturbance of the coracoid and its attached muscles; $(c)$ there is minimal splitting of the deltoid; $(d)$ there is a complete picture of the inside of the shoulder joint so that the humeral head, displaced labrum, and glenoid are all seen much more easily than by the anterior exposure.

VOI. $30 \mathrm{~B}$, NO. 1, FEBRUARY 1948 


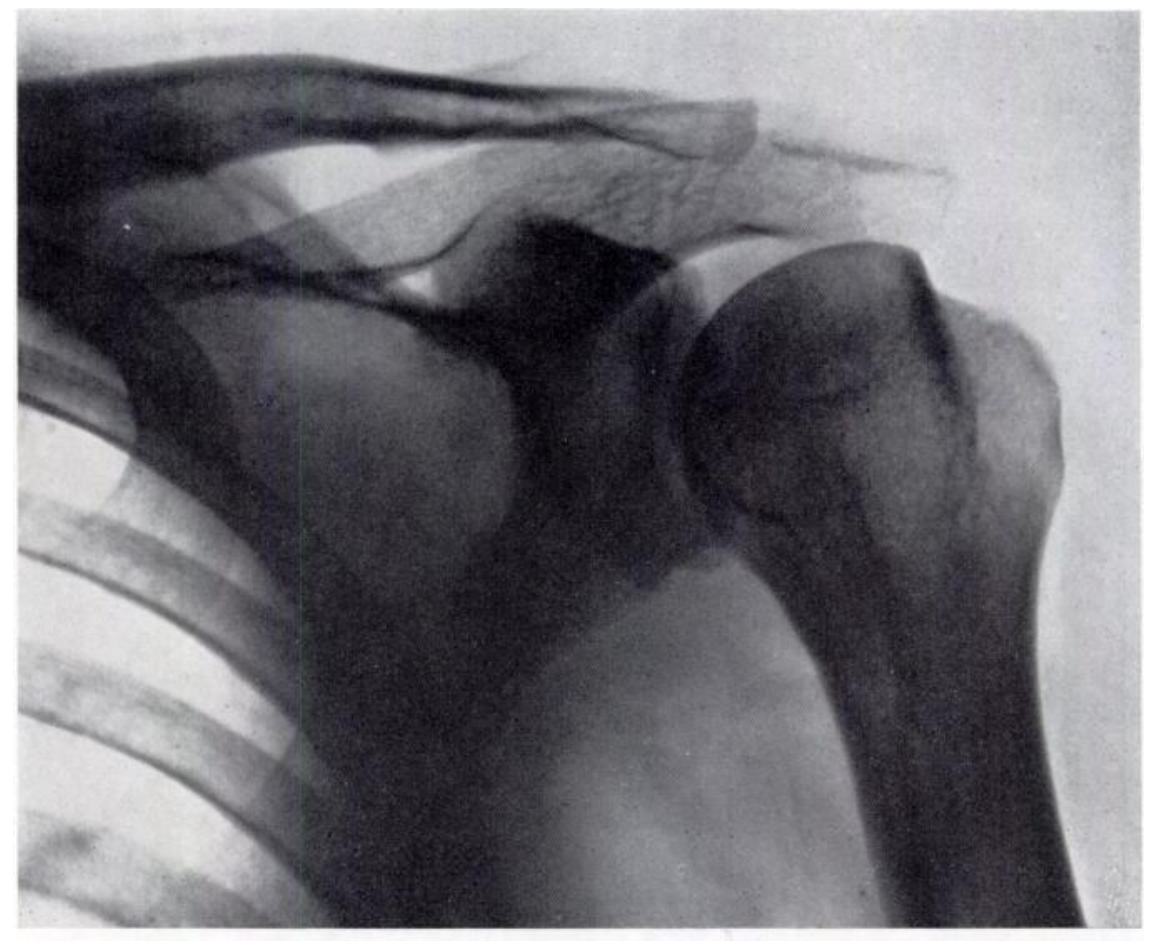

Fig. 4

When there is a large bone defect in the head of the humerus it is not enough to repair the labrial defect. Fxternal rotation movement must also be controlled in order to prevent engagement of the defective part of the humerus with the anterior glenoid margin.

(2) Sutures through the glenoid labrum and capsule are fixed with much greater ease and certainty, and with more complete visual control, than in the case of the anterior approach in which sutures are placed with some difficulty and uncertainty.

(3) We can now test from this case whether part of the required technique is fibrosis of the anterior capsule and tying-down of the subscapularis which has often been accredited in Bankart's operation.

The final words of McKecknie's operation-note are worthy of repetition. As the subsequent history showed, we were indeed in a position to test " whether part of the required technique is fibrosis of the anterior capsule and tying-down of the subscapularis." The labrial defect was repaired in this patient with even greater certainty than any other of the fifty-two cases. Yet in this case alone dislocation recurred within six months. In this case alone the anterior capsule had not been divided, reefed, double-breasted, or fibrosed. In this case alone the subscapularis had not been shortened or "tied-down." Despite the ease of exposure, and the facility of repair, the superior approach is clearly a mistake. It deals successfully with labrial detachments; but it fails to give sufficient control of external rotation movement to meet the problem of defects in the humeral head. The inference is obvious: Bankart's operation succeeds not alone by virtue of the repair of labrial lesions, but even more by virtue of the operative exposure of such lesions, and the subsequent resuture which limits external rotation movement. Bankart made a great contribution to surgery by relentlessly, bluntly and forcefully directing attention to the anterior margin of the gleno-humeral joint; but his success in the treatment of recurrent dislocation of the shoulder joint lies more in the operative exposure he advocated than in the "essential lesion" he described.

Summary-Fifty-two cases of exposure of the glenoid labrum are recorded. Fifty-one operations with anterior exposure, followed by capsular reefing and shortening of the subscapularis, were successful. One operation with superior exposure, and without capsular reefing or shortening of the subscapularis, was unsuccessful. 